
\title{
Psychische Gesundheit und Rückkehrvorstellungen am Beispiel von Flüchtlingen aus dem ehemaligen Jugoslawien
}

\author{
Ulrike von Lersner, Heide Rieder und Thomas Elbert
}

Zentrum für Psychiatrie Reichenau, Psychologische Ambulanz für Flüchtlinge

\begin{abstract}
Zusammenfassung. Theoretischer Hintergrund: Viele Flüchtlinge aus dem ehemaligen Jugoslawien leben seit ca. 11 Jahren in Deutschland. Es fehlt bislang an Daten zu den Langzeitfolgen der traumatischen Kriegsereignisse und der Einflüsse von Exilfaktoren auf die psychische Gesundheit in dieser Population. Auch sind Zusammenhänge zwischen diesen Faktoren sowie die Einstellung zur Rückkehr nicht ausreichend untersucht. Fragestellung: Wie ist die psychische Gesundheit dieser Flüchtlinge nach durchschnittlich 11 Jahren im Exilland Deutschland einzuschätzen? Welche Motive sprechen aus Sicht der Betroffenen für und gegen eine freiwillige Rückkehr ins Herkunftsland? Methoden: $n=50$ Flüchtlinge wurden zu ihrer aktuellen Lebenssituation, ihrer Einstellung zum Heimatland und zu freiwilliger Rückkehr befragt, sowie zu ihrer psychischen Gesundheit mit dem strukturierten Interview M.I.N.I. und den Fragebögen PDS und EUROHIS untersucht. $\quad$ Ergebnisse: Bei $78.0 \%$ liegt mindestens eine psychische Störung vor, es zeigen sich Zusammenhänge zwischen Lebenssituation in Deutschland, Rückkehrbereitschaft und psychischer Gesundheit. Schlussfolgerungen: Die psychische Belastung der Befragten ist sehr hoch, Ursachen hierfür sind sowohl die erhöhte Vulnerabilität auf Grund der Kriegsereignisse als auch die Belastung durch Postmigrationsfaktoren im Exil. Diese Faktoren sollten im Umgang mit Flüchtlingen in Deutschland berücksichtigt werden und haben auch Implikationen für die Planung von Rückkehrhilfeprogrammen.

Schlüsselwörter: Posttraumatische Belastungsstörungen, Psychische Gesundheit, Migration, Flüchtlinge
\end{abstract}

Mental health and attitudes towards returning to the country of origin in refugees from former Yugoslavia

\begin{abstract}
Background: Many refugees live for years in exile. The combination of stress in the host country, together with long-term effects of traumatic stress usually experienced in the home country, may affect mental health. Little is known about the extent to which these and other factors promote or prevent return to the country of origin. Here, we investigate refugees who came to Germany after the civil war on the Balkans and who have been living in exile for some 11 years. Objective: What is the mental health status of refugees who have been living in exile for an extended period? As we expected substantial suffering, we also asked what the motives for and reasons against a voluntary return to the country of origin? Methods: Fifty refugees were interviewed about their present living situation and about their views regarding their home country and voluntary return. The mental health of respondents was tested using the questionnaires PDS and EUROHIS and the structured interview M.I.N.I. Results: We found a prevalence rate of $78 \%$ for psychiatric disorders in the sample, a factor which prevented return to the country of origin. We also recorded substantial correlations between the living situation in Germany, disposition to return, and mental health. Conclusions: Psychological strain among the study participants were very high. Traumatic stress, experienced during war and in the host country, has left the victims vulnerable and not well equipped to cope with severe post-migration stressors in exile. These factors should be taken into account in programs for refugees, including those that offer support for return to the country of origin. Key words: posttraumatic stress disorder, mental health, migration, refugees
\end{abstract}

Zahllose kriegerische Konflikte treffen weltweit vornehmlich die Zivilbevölkerung und führen zu bleibend hohen Zahlen an Flüchtlingen und Asylsuchenden. Zusätzlich

Diese Studie wurde durchgeführt mit Unterstützung des Europäischen Flüchtlingsfonds (EFF) . Wir danken Frau Prof. Dr. Brigitte Rockstroh, Frau Dr. Margarethe Schauer und Frau Martina Ruf (Universität Konstanz) für Beratung und Unterstützung, den Koopera tionspartnern in den Flüchtlingsberatungsstellen und Rückführungsorganisationen, sowie den Flüchtlingen für ihre Teilnahme. verstärkt das globale ökonomische und ökologische Ungleichgewicht die Migration in die Industriestaaten, sodass die Wirtschafts- und Sozialsysteme der Zielstaaten insgesamt an die Grenzen ihrer Integrationsfähigkeit bzw. -willigkeit stoßen. Auf der Basis dieser Entwicklungen, aber auch aus wirtschaftlichen Überlegungen entwickelte sich in den letzten Jahren das Konzept der ,freiwilligen Rückführung“ zu einem der zentralen Instrumente der europäischen und auch der deutschen Migrationspolitik (BMI, 2001). Übersichten zu aktuellen Rückkehrprogram- 
men in Deutschland und Europa finden sich bei Entenmann (2002) und ZIRFcounselling (BAMF, 2006). Es bestehen große Unterschiede in der konkreten Umsetzung der nationalen und internationalen Programme. Grundsatz aller Programme ist jedoch die Freiwilligkeit der Entscheidung zur Ausreise und die Begleitung im Heimatland zur „humanitären Reintegration“ (nachzulesen in einem Positionspapier der Bundesarbeitsgemeinschaft der Freien Wohlfahrtspflege, 2006).

\section{Rückkehrmotive}

Vorhandene wissenschaftliche Arbeiten zu ,freiwilliger Rückkehr" beschäftigen sich vor allem mit den Rückkehrmotiven von Flüchtlingen und Migranten. Danach lassen sich Rückkehrmotive drei Dimensionen zuordnen: 1. ökonomische Gründe, 2. familiäre und persönliche Gründe und 3. patriotische und soziale Gründe (Gmelch, 1984). Eine andere häufige Form der Klassifikation ist die Unterteilung in so genannte „Pull- und Push-Faktoren“, welche sich jeweils in allen drei Dimensionen wieder finden (Haddon, zitiert in Lienenkamp, 1999). „Pull-Faktoren“ ziehen die Person aus dem Herkunftsland heraus an, wie etwa Familienbindungen, Heimweh und Loyalität zum Heimatland, während „Push-Faktoren“ solche Motive beschreiben, die die Person in Richtung Heimatland schieben, ihr den weiteren Aufenthalt im Gastland als unattraktiv erscheinen lassen. Diese sind etwa unzureichende finanzielle Mittel, unsicherer Aufenthaltsstatus, ungewohntes Klima und Diskriminierung (Powell et al., 1999; Dahinden, 2005). Studien haben mehrfach nachgewiesen, dass „Pull Faktoren“ größeren Einfluss haben, d. h. zentral für die Rückkehrentscheidung sind. Untersuchungen von Gmelch (1979), Taylor (1976) und Toren (1975) haben ergeben, dass in der Regel nicht nur ein einzigeGrund vorliegt, der für die Entscheidung zur Rückkehr verantwortlich ist. Vielmehr handelt es sich um ein Konglomerat an Gründen, von denen auf Nachfrage relativ willkürlich ein Motiv herausgegriffen wird. Dies dient u.a. dazu, die kognitive Dissonanz zwischen Pro- und Contra-Argumenten zu verringern, die für die meisten in einer solchen Entscheidung entsteht. Aus diesem Grund wurde in der vorliegenden Untersuchung eine Auswahl von mehreren Push- und Pull-Faktoren zusammengestellt, die sich den drei oben genannten Dimensionen zuordnen lassen.

\section{Psychische Gesundheit von Flüchtlingen}

Der durch Kriegs- und Gewalterfahrungen ausgelösten Posttraumatischen Belastungsstörung (PTSD) als eine in Flüchtlingspopulationen häufig diagnostizierte Störung wurde in jüngerer Zeit eine große Bedeutung zugesprochen (De Jong, 2001; Lopes Cardozo, 2003; Mollica, 2001; vgl. zur Übersicht Johnson \& Thompson, 2007). Bezogen auf Flüchtlinge aus dem ehemaligen Jugoslawien, die $\mathrm{Zu}-$ flucht im Exil gesucht haben, werden PTSD-Raten von 30-60\% (Thulesius \& Hakansson, 1999; Weine, 1998; Ai, 2002) berichtet. Der Anteil trauma-bedingter psychischer
Störungen und Funktionseinschränkungen variiert naturgemäß mit der Zahl traumatischer Stressoren und den politischen Lebensumständen in der befragten Population (Neuner et al., 2004; Miller et al., 2005; Gäbel et al., 2006; Maercker et al., in press), was die starke Variation der PTSD-Raten zwischen den einzelnen Studien erklärt.

Psychische Belastung wie seelische Erkrankungen sind bei Personen, die ihr Land verlassen hatten, häufig größer als bei Zurückgebliebenen oder intern Vertriebenen, wie etwa die Studie von Hunt und Gakenyi (2004) zeigt. Marshall und Kollegen (2005) wiesen nach, dass dieser Befund auch noch nach 20 Jahren im Exilland nachweisbar ist. Die große Aufmerksamkeit, die Patienten mit der Diagnose einer PTSD zukam, hatte in den letzten Jahren zur Folge, dass andere psychische Störungen in der allgemeinen Debatte wenig Beachtung fanden. Vermehrt vermuten jedoch verschiedene Autoren, dass neben traumatischen Erfahrungen postmigratorische Stressoren (wie Anpassungsschwierigkeiten, Verlust des kulturellen Kontexts und von Unterstützung, sowie die allgemeine Asylsituation) eine bedeutsame Rolle spielen könnten und zu anhaltenden psychischen Belastungen wie Angststörungen, Somatisierung und insbesondere Depression führen können (Kivling- Bodén \& Sundbom, 2002; Knipscheer \& Kleber, 2006). Den Einfluss der Exilsituation auf Entstehung und Aufrechterhaltung psychischer Auffälligkeiten untersuchten Steel et al. (1999) genauer und fanden, dass postmigratorische Faktoren 14.0\% der Varianz der PTSDSymptomatik im Vergleich zu 20. 0\% prämigratorischer Faktoren ausmachen. Miller und Kollegen (2002) fanden einen signifikanten Zusammenhang zwischen exilbezogenen Stressoren wie ein zu geringes Aktivitätsniveau oder soziale Isolation und depressiver Symptomatik bei bosnischen Flüchtlingen. Lehmann (2006) beobachtete in einer Längsschnittstudie, dass sich bei Gewährung eines sicheren Aufenthaltsstatus depressive Symptome verbesserten, nicht jedoch diejenigen einer PTSD.

\section{Psychische Gesundheit von Rückkehrern}

Zur psychischen Gesundheit von Rückkehrern sind bisher wenige Publikationen erschienen. Sundquist et al. (1996) begleiteten Flüchtlinge aus Chile und Uruguay und fanden bei den zurückgekehrten Personen niedrigere Werte für psychische Gesundheit und Integration als bei den im Exil Verbliebenen. Toscani et al. (2006), die die Lebensbedingungen der aus der Schweiz zurückgekehrten Kosovoalbaner untersuchten, fanden $25.5 \%$ PTSD unter den Befragten, eine Kontrollgruppe in der Schweiz wurde leider nicht erhoben. $65.0 \%$ der Rückkehrer lebten in extremer Armut und wiesen sehr geringe Werte für allgemeine Gesundheit auf. Des Weiteren fanden die Autoren einen negativen Zusammenhang zwischen Verweildauer im Exil und psychischer Gesundheit nach der Rückkehr. Roth et al. (2006) untersuchten Flüchtlinge aus dem Kosovo kurz nach ihrer Ankunft in Schweden, sowie 3, 6 und 18 Monate später an ihren jeweiligen Aufenthaltsorten. Bei Ankunft wurde bei 37.0\% der Flüchtlinge eine PTSD festgestellt. Nach 18 Monaten war ein Teil der Personen wieder 
in den Kosovo zurückgekehrt, die PTSD-Prävalenz lag in dieser Gruppe bei $52.0 \%$, während sie bei den in Schweden verbliebenen Flüchtlingen auf $87.0 \%$ angestiegen war. Höhere psychische Belastungen bei im Exil verbliebenen Flüchtlingen wird in dieser Studie mit zusätzlichen Be- lastungen durch Postmigrationsfaktoren im Exil erklärt (Silove, 1998, 2001), womit jedoch ein Widerspruch zu den in Abschnitt „Psychische Gesundheit von Flüchtlingen“ dargestellten Befunden entsteht, dem in zukünftigen Untersuchungen nachgegangen werden sollte.

Tabelle 1. Soziodemographische Daten der befragten Flüchtlinge, $n=50^{1}$

\begin{tabular}{|c|c|c|c|c|c|c|c|c|}
\hline \multirow{2}{*}{$\begin{array}{l}\text { Variable } \\
\text { Alter }\end{array}$} & \multirow[b]{2}{*}{$M(S D)$} & & \multicolumn{2}{|c|}{$\begin{array}{l}\text { Gesamt } \\
(n=50)\end{array}$} & \multicolumn{2}{|c|}{$\begin{array}{l}\text { PTSD } \\
(n=27)\end{array}$} & \multicolumn{2}{|c|}{$\begin{array}{l}\text { ohne PTSD } \\
(n=23)\end{array}$} \\
\hline & & & 39.3 & $(9.9)$ & 39.8 & $(9.9)$ & 38.7 & $(9.9)$ \\
\hline \multirow[t]{2}{*}{ Geschlecht } & $N(\%)$ & Männlich & 23 & $(46.0)$ & 9 & $(33.3)$ & 14 & $(60.9)$ \\
\hline & & Weiblich & 27 & $(54.0)$ & 18 & $(66.7)$ & 9 & (39.1) \\
\hline \multirow[t]{3}{*}{ Herkunftsland } & $N(\%)$ & Bosnien & 21 & $(42.0)$ & 15 & $(55.6)$ & 6 & $(26.1)^{2}$ \\
\hline & & Serbien & 10 & (20.0) & 5 & (18.5) & 5 & (21.7) \\
\hline & & Kosovo & 19 & $(38.0)$ & 7 & $(25.9)$ & 12 & $(52.2)$ \\
\hline \multirow[t]{5}{*}{ Ethnie } & $N(\%)$ & Bosnisch & 19 & $(38.0)$ & 13 & $(48.1)$ & 6 & $(26.1)$ \\
\hline & & Serbisch & 4 & $(8.0)$ & 4 & $(14.8)$ & 0 & $(4.3)$ \\
\hline & & Albanisch & 13 & $(26.0)$ & 4 & (14.8) & 9 & (39.1) \\
\hline & & Roma & 13 & (26.0) & 6 & $(22.2)$ & 7 & $(30.4)$ \\
\hline & & Andere & 1 & $(2.0)$ & - & & 1 & $(4.3)$ \\
\hline \multirow[t]{4}{*}{ Familienstand } & $N(\%)$ & Ledig & 10 & $(20.0)$ & 6 & $(22.2)$ & 4 & (17.4) \\
\hline & & Verheiratet & 27 & $(54.0)$ & 13 & $(48.1)$ & 14 & $(60.9)$ \\
\hline & & Geschieden/Getrennt & at 8 & $(16.0)$ & 5 & (18.5) & 3 & (13.0) \\
\hline & & Verwitwet & 5 & $(10.0)$ & 3 & (11.1) & 2 & $(8.7)$ \\
\hline \multirow{2}{*}{$\begin{array}{l}\text { Aufenthalt in D. } \\
\text { in Jahren }\end{array}$} & $M(S D)$ & & 11.3 & (3.2) & 10.6 & (3.5) & 12.0 & $(2.8)$ \\
\hline & $\operatorname{Min}(\operatorname{Max})$ & & 3 & (16) & 3 & (14) & 7 & (16) \\
\hline \multirow{3}{*}{$\begin{array}{l}\text { Kinder in } \\
\text { Ausbildung in D. }\end{array}$} & $N(\%)$ & $\mathrm{Ja}$ & 31 & $(62.0)$ & 18 & $(66.7)$ & 13 & $(56.5)$ \\
\hline & & Nein & 6 & $(12.0)$ & 2 & $(7.4)$ & 4 & (17.4) \\
\hline & & Ohne Kinder & 13 & $(26.0)$ & 7 & $(25.9)$ & 6 & (26.1) \\
\hline \multirow[t]{4}{*}{ Jahre an Bildung } & $N(\%)$ & $0-6$ & 14 & $(28.0)$ & 8 & (29.6) & 6 & $(26.0)$ \\
\hline & & $7-10$ & 16 & $(32.0)$ & 10 & $(37.0)$ & 6 & $(26.0)$ \\
\hline & & $11-18$ & 20 & $(40.0)$ & 9 & (33.3) & 11 & $(47.7)$ \\
\hline & & Mittelwert & 9.2 & $(4.1)$ & 8.7 & $(3.0)$ & 9.8 & $(5.0)^{3}$ \\
\hline \multirow[t]{3}{*}{ Beschäftigung in D. } & $N(\%)$ & Arbeitend & 25 & $(50.0)$ & 12 & (44.4) & 11 & $(47.8)$ \\
\hline & & Arbeitslos & 23 & (46.0) & 13 & $(48.1)$ & 12 & $(52.2)$ \\
\hline & & Unbekannt & 2 & $(4.0)$ & 2 & & - & \\
\hline \multirow[t]{3}{*}{ Arbeitserlaubnis } & $N(\%)$ & $\mathrm{Ja}$ & 21 & $(42.0)$ & 11 & (40.7) & 10 & $(52.2)$ \\
\hline & & Nein & 28 & $(56.0)$ & 16 & (59.3) & 12 & (43.5) \\
\hline & & Unbekannt & 1 & $(2.0)$ & - & & 1 & \\
\hline \multirow{3}{*}{$\begin{array}{l}\text { Aufenthaltsstatus } \\
\text { (alte Bezeichnung) }\end{array}$} & $N(\%)$ & Unsicher & 34 & $(68.0)$ & 17 & (63.0) & 17 & (73.9) \\
\hline & & Befristet & 11 & (22.0) & 9 & (33.3) & 2 & $(8.7)$ \\
\hline & & Unbefristet & 5 & $(10.0)$ & 1 & $(3.7)$ & 4 & (17.4) \\
\hline
\end{tabular}

Anmerkungen: ${ }^{1}$ Mit einigen Personen konnten aus Gründen der hohen psychischen oder emotionalen Belastung nicht alle Abschnitte des Interviews durchgeführt werden, weshalb sich die Angaben in Tabelle 1 bei einigen Werten auf $n<50$ beziehen. ${ }^{2}$ Fishers Exact, $p<.05$. ${ }^{3} t(48)=$ $8.20, p<.05$. 


\section{Ziel der Studie und Besonderheit der untersuchten Stichprobe}

Erklärtes Ziel von Rückkehrmaßnahmen ist neben dem Bemühen, auf diese Weise zur Lösung nationaler Flüchtlingsfragen beizutragen, immer die nachhaltige humanitäre Reintegration in das Heimatland. Bislang liegen jedoch in Deutschland keine wissenschaftlichen Daten zur Nachhaltigkeit dieser Maßnahmen bzw. zu den Auswirkungen der Rückkehr auf die psychische Gesundheit der betroffenen Personen vor. Die wenigen vorhandenen Studien zur psychischen Gesundheit von Rückkehrern liefern zudem widersprüchliche Ergebnisse.

Die vorliegende Untersuchung stellt den ersten Abschnitt einer Längsschnittstudie dar, die zum Ziel hat, das Konzept „freiwillige Rückkehr“ unter psychologischen Gesichtspunkten zu analysieren. Dieser erste Abschnitt analysiert die Situation von Personen, die im Rahmen des Krieges auf dem Balkan vor ca. 11 Jahren nach Deutschland geflüchtet waren und nicht zurückkehren möchten. Untersucht wurden aktuelle Lebenssituation, Einstellung zur ,freiwilligen Rückkehr“, psychische Gesundheit und subjektive Lebenszufriedenheit.

Es sei darauf hingewiesen, dass es sich dabei um eine besondere Gruppe der aus dieser Region nach Deutschland Geflüchteten handelt. Während die überwiegende Mehrheit ohne staatliche Hilfe freiwillig zurückkehrt oder in andere Länder emigriert ist, sind diejenigen geblieben, die entweder eine dauerhafte Aufenthaltsgenehmigung erhielten, krankheitsbedingt Aufenthalt gewährt bekamen oder aber von jahrelangen „Kettenduldungen“ betroffen waren, wenn so genannte Abschiebehindernisse vorlagen (BAMF, 2007). Untersucht wird von daher auch der Einfluss einiger Stressoren im Exil sowie der Grad der Integration.

\section{Methodik}

\section{Stichprobe}

50 Personen aus dem Gebiet des ehemaligen Jugoslawien, die im Laufe der 90er Jahre infolge des Bürgerkrieges nach Deutschland geflohen sind, wurden von geschulten Mitarbeitern der Psychologischen Modell- und Forschungsambulanz der Universität Konstanz befragt. In Tabelle 1 sind die sozialen und demographischen Kennwerte der Befragten dargestellt.

\section{Durchführung}

Für die Untersuchung wurden bundesweit 21 Flüchtlingszentren, Kulturzentren, Rechtsberatungsstellen, Sprachkurse und Arztpraxen kontaktiert.

Einige Institutionen und Flüchtlinge lehnten eine Teilnahme an der Studie ab, da sie das politische Instrument der „freiwilligen Rückkehr“ per se ablehnen. Einige Flüchtlinge verweigerten eine Teilnahme, weil Ängste bestanden, erneut über das Thema der Flucht und des Krieges sprechen zu müssen. Andere befürchteten einen negativen Einfluss auf ihr Aufenthaltsverfahren, wenn sie über eine Teilnahme an der Studie mit dem Thema der, ,freiwilligen Rückkehr" in Verbindung gebracht würden. Die untersuchte Stichprobe setzt sich zusammen aus Teilnehmern aus 7 der 11 kontaktierten Institutionen, in denen sich wiederum etwa die Hälfte der angesprochenen Probanden $(N=108)$ zu einer Teilnahme an der Studie bereit erklärte.

Die Interviews fanden je nach Wunsch der Befragten entweder in den Wohnräumen derselben oder in den Räumen der genannten Träger statt. Sie wurden von Mitarbeitern der Psychologischen Modell- und Forschungsambulanz der Universität Konstanz durchgeführt. Als Sprachmittler wurden Personen eingesetzt, welche bereits über Erfahrung in der Übersetzungsarbeit mit der untersuchten Klientel verfügten und zusätzlich zu den Inhalten der vorliegenden Studie geschult worden waren. Ein Interview dauerte ca. 2 Stunden. Darin wurden auch alle Fragebögen in mündlicher Form abgefragt. Die Teilnahme an der Untersuchung war freiwillig, ein Abbruch des Gesprächs war auf Wunsch zu jedem Zeitpunkt möglich, wurde jedoch nicht in Anspruch genommen. Alle Teilnehmer erhielten eine schriftliche Schweigepflichtserklärung von Seiten der Interviewer und der Sprachmittler.

Wurde bei einer Person eine psychische Störung festgestellt und befand sich diese noch nicht in Behandlung, so erhielt diese eine kurze Psychoedukation sowie Kontaktadressen von psychosozialen Einrichtungen im Umkreis des Wohnortes.

\section{Messinstrumente}

Soziodemographischer Rückkehr-Fragebogen : Der soziodemographische Fragebogen dient dazu, Informationen über den Lebenshintergrund der Probanden zu erhalten. Er enthält Fragen zu den Themenkomplexen Herkunft und Religion, Alter, Geschlecht, Familienstatus, Bildungsstand und Beschäftigungssituation, zu Fluchtgründen, zu Aufenthalt und Integration in Deutschland, zum Bezug zum Herkunftsland und zur klinischen Vorgeschichte. Der Integrationsstatus wird hier theoretischen Vorüberlegungen zufolge (Heckmann, 2003) als Konglomerat aus verschiedenen Aspekten wie Aufenthaltstitel, Arbeitserlaubnis und Beschäftigungssituation (strukturelle Ebene), Freizeitverhalten und Kontakte zu Deutschen bzw. eigenen Landsleuten (soziale Ebene) und Deutschkenntnisse sowie das Zugehörigkeitsgefühl (kulturelle Ebene) verstanden. Inhaltlich wird die Antwort für jeden Aspekt auf einer 3-stufigen Skala von 0 (keine Integration), 1 (geringe) bis 2 (gute) bewertet und in der Auswertung als Summenscore verwendet.

Ein weiterer Abschnitt widmet sich den Motiven, die für und gegen ein Verbleiben in Deutschland bzw. für und 
gegen eine freiwillige Rückkehr in das Herkunftsland sprechen. Sie lassen sich auf den drei Dimensionen von Gmelch (1984) abbilden.

M.I.N.I.: Die deutsche Version des Mini International Neuropsychiatric Interview, Version 5.0.0 (Sheehan et al., 1998, deutsche Übersetzung: Ackenheil et al.,1999) ist ein kurzes strukturiertes Interview zur Erstellung von Diagnosen bei klinischen Störungen auf Basis des DSM-IV. Dieses Instrument weist sowohl eine hohe Konkordanz mit ausführlichen strukturierten Interviewinstrumenten wie CIDI (WHO, 1990) oder SKID (Spitzer et al., 1990) auf, als auch eine hohe Sensitivität und Spezifität (Sheehan et al., 1998). In der vorliegenden Untersuchung wurden die Abschnitte I (Posttraumatische Belastungsstörung), L (Psychotische Störungen) und P (Antisoziale Persönlichkeitsstörung) nicht erhoben.

EUROHIS Quality of Life Scale : Dieses Instrument dient zur Erhebung der subjektiven Einschätzung der Lebensqualität und stellt eine Kurzform des WHOQOLBREF (WHOQOL Group, 1998) dar. Es enthält 8 Fragen zur Zufriedenheit mit der eigenen Person sowie verschiedenen Lebensbereichen. Jede Frage kann auf einer Skala von 1 (sehr gut) bis 5 (sehr schlecht) beantwortet werden. Die Summe der Punktwerte geteilt durch die Anzahl der Unterfragen ergibt den Zufriedenheitsindex pro Person. Eine repräsentative Untersuchung in 10 Ländern konnte gute kulturübergreifende Einsetzbarkeit und zufrieden stellende Werte für die konvergenten und diskriminanten Validitätswerte nachweisen (Schmidt et al., 2006).

Posttraumatic Stress Diagnostic Scale (PDS; Foa et al., 1995; deutsche Übersetzung: Ehlers, Steil \& Winter, 1995) ist ein Selbstberichtsfragebogen, der als ScreeningInstrument eingesetzt werden kann und mit dessen Hilfe sowohl Hinweise auf das Vorliegen einer PTSD, als auch auf deren Schweregrad erhoben werden können. Er setzt sich zusammen aus einem Ereignisteil, in dem die erlebten traumatischen Ereignisse systematisch erhoben werden und einem zweiten Teil, in dem die Symptome in enger Anlehnung an den DSM-IV ermittelt werden. Diese Screening-Intrument weist sowohl eine hohe Interraterreliabilität, als auch eine hohe Spezifität und Sensitivität auf (Foa, 1995).

\section{Datenanalyse}

Die Datenanalyse wurde mit Hilfe des Statistikprogramms SPSS ® 11.5 für Windows durchgeführt. Es wurden die Häufigkeiten des Auftretens der einzelnen psychischen Störungen sowie die Mittelwerte der Angaben zur allgemeinen subjektiven Lebenszufriedenheit erhoben. Indizes zum Integrationsgrad wurden gemäß der Darstellung in „Methoden“ ermittelt. Gruppenunterschiede zwischen PTSD-Betroffenen vs. Nicht-Betroffenen bzw. psychisch Erkrankten und Gesunden bezüglich der untersuchten Konstrukte wurden mit zweiseitigen $t$-Tests für unabhängige Stichproben bzw. den exakten Test nach Fisher für kleine Stichproben sowie Chi-Quadrat-Tests bzw. MannWhitney-U-Tests analysiert. Die Signifikanzangaben für Gruppenunterschiede wurden einer Bonferoni-Korrektur unterzogen. Zusammenhangsmaße wurden mit Hilfe des Rangkorrelationskoeffizienten $\rho$ für ordinales Datenniveau nach Spearman errechnet und werden im Folgenden mit $r$ bezeichnet. Des Weiteren wurden logistische und lineare Regressionsanalysen durchgeführt, um zentrale Einflussfaktoren auf die psychische Gesundheit zu ermitteln.

\section{Ergebnisse}

\section{Psychische Gesundheit}

Die Untersuchungen ergaben eine hohe Punktprävalenz psychischer Störungen unter den befragten Flüchtlingen. Bei 39 der 50 Personen (78.0\%) wurde zum Zeitpunkt der Befragung mindestens eine psychische Störung nach DSM-IV diagnostiziert. Wie Tabelle 2 zeigt, war PTSD mit einer Prävalenz von 54.0 \% am häufigsten vertreten, gefolgt von affektiven Störungen und Angststörungen, welche entweder komorbid zur PTSD oder eigenständig auftraten.

Es zeigte sich ein negativer Zusammenhang zwischen Bildungsgrad - gemessen in Jahren an Bildung - und psychischer Gesundheit $(r=-.34, p<.05)$.

Die Suizidalität war allgemein hoch. In der PTSD-Gruppe war sie jedoch noch einmal signifikant höher als in der Gruppe der Nicht-Betroffenen $\left(C h i^{2}(3)=9.21, p<.05\right)$.

Ausschlaggebend für die Herausbildung einer PTSD waren in den meisten Fällen Kriegserlebnisse (33.3\%) und sexuelle Gewalt (25.9\%). Unabhängig von der Diagnose einer PTSD berichteten die Befragten eine große Anzahl traumatischer Ereignisse. Besonders häufig wurden hierbei Ereignisse im Zusammenhang mit Gewalterleben gegen die eigene Person (54. 0\%), gegen ein Familienmitglied (62.0\%) und Aufenthalt im Kriegsgebiet (64.0\%) genannt.

Es fand sich ein negativer Zusammenhang zwischen PTSD und dem Interesse, das Herkunftsland zu besuchen $(r=-.28, p<.05)$ sowie Kontakte zu Freunden und Familienmitgliedern in der Heimat zu pflegen $(x=-.37, p<.01)$.

$46.0 \%$ der Befragten befanden sich bereits in psychiatrischer (12. $0 \%)$ und/oder psychotherapeutischer (52.0\%) Behandlung, davon 77.8\% aus der PTSD-Gruppe.

Abschließend wurden mithilfe linearer bzw. logistischer Regressionsanalysen die wichtigsten Zusammenhänge im Rahmen psychischer Gesundheit ermittelt. Diese sind für PTSD: $\beta_{\text {(Anzahl traumat. Ereignisse) }}=.53(p<.01)$, für affektive Störungen: $\beta_{\text {(Integration) }}=-.39(p<.05)$ und für Integration: $\beta_{(\text {psychische Erkrankungen })}=-1.67(p<.01)$. 
Tabelle 2. Psychische Gesundheit

\begin{tabular}{|c|c|c|c|c|c|c|}
\hline \multirow[b]{2}{*}{ Erkrankung/Störung } & \multicolumn{2}{|c|}{ Gesamtstichprobe } & \multicolumn{2}{|c|}{ Mit PTSD } & \multicolumn{2}{|c|}{ Ohne PTSD } \\
\hline & $N$ & $\%$ & $N$ & $\%$ & $N$ & $\%$ \\
\hline PTSD & 27 & 54.0 & 27 & 100.0 & - & \\
\hline Depression & 25 & 50.0 & 17 & 62.9 & 8 & 36.4 \\
\hline Dysthymie & 7 & 14.0 & 5 & 18.5 & 2 & 9.1 \\
\hline Suizidalität & 21 & 32.0 & 16 & 59.2 & 5 & 22.7 \\
\hline Manie & 1 & 2.0 & 1 & 3.7 & - & \\
\hline Panik aktuell & 5 & 10.0 & 4 & 14.8 & 1 & 4.5 \\
\hline Agoraphobie aktuell & 5 & 10.0 & 4 & 14.8 & 1 & 4.5 \\
\hline Soziale Phobie & 5 & 10.0 & 4 & 14.8 & 1 & 4.5 \\
\hline Zwangsstörung & - & & - & & - & \\
\hline Substanzabhängigkeit & - & & - & & - & \\
\hline Substanzmißbrauch & 2 & 4.0 & 1 & 3.7 & 1 & 4.5 \\
\hline Anorexia Nervosa & - & & - & & - & \\
\hline Bulimia Nervosa & 2 & 4.0 & 2 & 7.4 & - & \\
\hline Generalisierte Angst & - & & - & & - & \\
\hline \multicolumn{7}{|l|}{ Behandlung beim } \\
\hline Psychologen/Psychiater & 29 & 58.0 & 21 & 77.8 & 8 & 36.4 \\
\hline \multicolumn{7}{|l|}{ Therapieerfahrung: } \\
\hline • Einzel & 10 & 20.0 & 8 & 29.6 & 2 & 9.0 \\
\hline - Gruppe & 13 & 26.0 & 8 & 29.6 & 5 & 22.7 \\
\hline
\end{tabular}

\section{Subjektive Lebenszufriedenheit}

Die durchschnittliche subjektive Lebenszufriedenheit lag in der Untersuchungsgruppe auf einer Skala von 1-5 bei $m=3.16(S D=.83)$. Personen mit PTSD gaben mim $=2.90$ $(S D=.60)$ einen signifikant geringeren Durchschnittswert an als Personen mit anderen psychischen Erkrankungen, bei denen der Wert für die subjektive Lebensqualität bei $m=3.45(S D=.96)$ lag $(p<.05)$. Bei Gesunden lag er bei $m=4.03(S D=.59)$. Die statistische Analyse der Daten ergab einen negativen Zusammenhang zwischen subjektiver Lebenszufriedenheit und psychischer Gesundheit im Allgemeinen $(r=-.61, p<.01)$, sowie Posttraumatischen Belastungsstörungen im Speziellen $(r=-.36, p<.05)$.

\section{Integration}

Der Integrationsindex in der Untersuchungsgruppe lag auf einer Werteskala von $0-12$ beim $=6.49(S D=2.29)$. Bei Personen mit psychischen Erkrankungen lag er bei $m=$ $6.64(S D=1.75)$, bei PTSD-Betroffenen beim $=6.29(S D=$ 1.85). Für psychisch Gesunde ergab sich ein durchschnittlicher Wert von $m=7.83(S D=2.55)$.

Psychisch Gesunde und Kranke unterschieden sich im Grad ihrer Integration signifikant $(42.0)=2.86, p<.01)$, die Gruppe der PTSD-Betroffenen unterschied sich hierin nicht signifikant von anderen psychisch Erkrankten $\mathbf{~ ( 4 2 . 0 )}$ $=1.09, p>.05$ ).

Es zeigte sich ein Zusammenhang zwischen subjektiver Lebenszufriedenheit und Integration $(x=.40, p<.01)$.
Ebenso ließ sich ein negativer Zusammenhang zwischen psychischer Gesundheit und Integration $(x=-.34, p<.05)$ nachweisen.

\section{Fluchtgründe und Rückkehrmotive}

Gebeten, auf einer Skala von 1 (gar nicht) bis 10 (sehr hoch) anzugeben, wie ausgeprägt die Bereitschaft sei, unter den gegebenen Lebensumständen freiwillig in die Heimat zurückzukehren, gaben $76.0 \%$ der Befragten den Wert 1 an, dem Bereich oberhalb des Wertes 5 ordneten sich $8.0 \% \mathrm{zu}$. Als Gründe, die für eine Rückkehr in das Heimatland sprachen, wurden familiäre Bindungen genannt, gefolgt von Arbeits- und Perspektivlosigkeit in Deutschland. Gegen eine Rückkehr sprach die Anbindung an Deutschland, die in erster Linie über die Ausbildung und damit verbundenen besseren Zukunftsperspektiven für die Kinder in Deutschland definiert wurde. Dieses Motiv, welches sich der Contra-Seite zuordnen lässt, wurde in der Gesamtgruppe als häufigstes - und nach Aussage der Probanden entscheidendes - Motiv zum Thema „freiwillige Rückkehr“ angegeben.

In der PTSD-Gruppe wurde als Hauptgrund gegen eine Rückkehr die Angst vor der Wiedererinnerung an traumatische Erlebnisse in der Heimat berichtet und ein signifikanter Zusammenhang zwischen PTSD und diesem Rückkehrmotiv gefunden $(r=.39, p<.01)$, gefolgt von der besseren medizinischen Versorgung in Deutschland und ebenfalls der Anbindung an Deutschland über die eigenen Kinder. 


\section{Diskussion}

\section{Psychische Gesundheit}

In Anbetracht der Befunde der vorliegenden Untersuchung ist die psychische Belastung unter Flüchtlingen aus dem ehemaligen Jugoslawien, die nach 11 Jahren weiterhin in Deutschland leben, alarmierend hoch. Es kann davon ausgegangen werden, dass die Stichprobe repräsentativ für die noch in Deutschland verbliebenen Flüchtlinge aus dem ehemaligen Jugoslawien ist, da die Befragten aus einem weiten Spektrum öffentlicher Aufenthaltsorte für die Studie gewonnen wurden. Eingangs sprachen wir von einer besonderen Gruppe von Flüchtlingen aus dem ehemaligen Jugoslawien, die sich heute noch in Deutschland aufhalten. Neben denen, die den Flüchtlingsstatus abgelegt haben und sich häufig gut in die deutsche Gesellschaft integrieren konnten, sind es nicht selten kranke oder anderweitig geschwächte Menschen, die weiterhin mit diesem eigentlich temporären Status in Deutschland leben. Wie bereits in früheren Studien an ähnlichen Populationen gezeigt werden konnte, treten unter den Teilnehmern insbesondere affektive und Angststörungen auf, PTSD spielt eine übergeordnete Rolle. Aus geschichtlichen Zusammenhängen ergibt sich die hohe Zahl der Bosnier unter den PTSD-Betroffenen. Alarmierend ist die hohe Rate an Suizidalität unter den Befragten. Die Hälfte aller Befragten befand sich in psychologischer Behandlung, was für eine gute Versorgung der Flüchtlinge spricht. In einigen Region Deutschlands ist jedoch die Verlängerung des Aufenthaltes an eine Behandlung gekoppelt. Dies hat unter Umständen in einigen Fällen dazu geführt, sich eher aus diesen Gründen als aus eigener Überzeugung in Behandlung zu begeben. Ungeachtet dessen ist bei einer derartig hohen Suizidalitätsrate eine psychologische Behandlung dringend geboten. Interessant ist der positive Einfluss von Bildung auf die psychische Gesundheit. Wie bereits in früheren Untersuchungen nachgewiesen (Herman, 1994; Kleck, 2006), scheint es sich hierbei um einen protektiven Faktor zu handeln.

Eine große Gruppe der Befragten berichtete von traumatischen Erlebnissen im Kontext des Krieges und ihrer Flucht. Daraus resultierend hat sich bei einem großen Teil der Befragten eine PTSD herausgebildet.

Als weitere Einflussfaktoren für psychische Erkrankungen müssen nach ca. 11 Jahren im Exil Postmigrationsfaktoren herangezogen werden. Faktoren, die in der vorliegenden Untersuchung erneut als derartige Exilstressoren bestätigt wurden, sind unsicherer Aufenthaltsstatus, fehlende Arbeitserlaubnis und mangelnde Sprachkenntnisse. Sie erwiesen sich als ausschlaggebend in der Herausbildung von affektiven Störungen in der untersuchten Population. Nicht auszuschließen ist, dass einige der Flüchtlinge auch unabhängig von Traumafolgestörungen bereits mit psychischen Erkrankungen nach Deutschland eingereist sind. Solche Personen hatten zum einen nicht die Ressourcen, in ihr Heimatland zurückzukehren, gleichzeitig bietet ihnen das medizinische System der Bundesre- publik bessere Behandlungsmöglichkeiten, was auch als Motiv gegen eine Rückkehr erwähnt wurde.

\section{Integration}

Eine schlechte Integration ging insgesamt mit einer höheren psychischen Belastung und einer geringeren subjektiven Lebensqualität einher. Wie bereits dargestellt, hatte eine geringe Integration Einfluss auf die Entwicklung von affektiven Störungen, welche in der Untersuchungsgruppe insgesamt am häufigsten diagnostiziert wurden. Dieses Ergebnis entspricht bereits existierenden Studien an Flüchtlinge im Exil (Silove, 1998). Vermutlich erhöhen fehlende Integration und zusätzliche Exilstressoren das Risiko für psychische Anfälligkeit. In der Folge führen psychische Erkrankungen zu sozialem Rückzug, erschweren das Erlernen einer Sprache und behindern damit die Integration zusätzlich. Als ein zentrales Integrationshindernis ist ein unsicherer Aufenthaltsstatus zu nennen, da er nach geltendem Aufenthaltsrecht einer Integration diametral entgegensteht. Wird die Unsicherheit bezüglich der eigenen Zukunft zum Dauerzustand, kann es zu einem Phänomen kommen, was in der Literatur als „Wartesaalposition“ oder „Abhängigkeitssyndrom“ bezeichnet wurde. (Buchwald, zitiert in Emminghaus et al., 2003). Aus anfänglich normalen emotionalen Reaktionen wie Besorgtheit oder Enttäuschung können so im zeitlichen Verlauf psychische Störungen mit Krankheitswert werden. Die vorliegende Untersuchung gibt Hinweise darauf, indem sich eine psychische Erkrankung als wichtiger Einflussfaktor für eine schlechte Integration zeigte.

Ein Zusammenhang zwischen Beschäftigung und psychischer Gesundheit konnte nicht nachgewiesen werden. Beschäftigung - unabhängig davon, ob es sich um legale oder illegale Arbeit handelte - stand jedoch in positivem Zusammenhang zu Integration und subjektiver Lebenszufriedenheit und wirkte sich darüber möglicherweise indirekt positiv auf die psychische Gesundheit aus.

Zum aktuellen Zeitpunkt liegt im deutschen Sprachraum kein validiertes Instrument zur Messung von Integration vor. Erste Versuche zur Schaffung solcher Indikatoren und Skalen machen Gesemann (2006) und Michalowski und Snel (2005), die sich ebenfalls auf die Definitionen von Heckmann (2003) stützen. In beiden Projektgruppen ist jedoch die Operationalisierung des Konstrukts "Integration“ nicht abgeschlossen, weshalb sich auch die vorliegende Studie an den prominenten theoretischen Konzepten orientiert, ohne ein validiertes Messinstrument anwenden zu können.

\section{Subjektive Lebenszufriedenheit}

Trotz besorgniserregender Werte bei psychischer Gesundheit und Lebenssituation sahen sich die Flüchtlinge hinsichtlich ihrer subjektiven Lebenszufriedenheit auf ähnlichem Niveau wie die Normalbevölkerung. In einer kulturübergreifenden Studie von Schmidt und Kollegen 
(2006) an gesunden Probanden aus 10 Nationen lag der Wert von $m=3.68(S D=0.62)$ nur unwesentlich höher. Möglicher Erklärungsansatz ist, dass aufseiten der Flüchtlinge ein Vergleich nach unten geschieht, d.h. Flüchtlinge ihre Situation mit den vermeintlich schlechteren Lebensbedingungen im Herkunftsland vergleichen. Betrachtet man objektive Faktoren wie Wohnsituation, soziale Sicherung und medizinische Versorgung, ist diese Einschätzung häufig vermutlich auch realistisch. Viele Flüchtlinge erwähnten im Laufe der Interviews auch immer wieder die große Dankbarkeit gegenüber Deutschland, das sie über solch lange Zeit hinweg unterstütze. Zumindest anteilig ist anzunehmen, dass dieser Aspekt das Ergebnis mit beeinflusst hat.

\section{Rückkehrmotive}

Als Hauptmotiv zum Thema „freiwillige Rückkehr“" wurde von den Befragten die Anbindung an Deutschland über die eigenen Kinder genannt, die den Entschluss zur Folge hatte, nicht in die Heimat zurückkehren zu wollen. Die Befragten gaben nahezu durchgehend an, nach den belastenden Erfahrungen des Krieges, der Flucht und der durchschnittlich 11 Jahre im Exil selbst keine Zukunftspläne mehr zu haben. Stattdessen richte sich ihr Streben jetzt allein auf die Zukunft und das Wohl der Kinder. Die Mehrheit der suizidalen Personen gab dies auch als Motiv an, den Suizidgedanken nicht nachzugeben. Hinzu komme, dass sich die Heimat in der Zwischenzeit verändert habe und vor allem von den Kindern, jedoch auch von den Erwachsenen als Fremde erlebt werde. Es hat im Laufe der Zeit eine Verschiebung stattgefunden von ursprünglich politisch motivierten Fluchtgründen hin zu ökonomischen und persönlichen Gründen, die als Rückkehrhindernisse angegeben werden. An dieser Stelle sei auf die oben bereits zitierte „Wartesaalposition“ hingewiesen, die die Flüchtlinge über Jahre daran hinderte bzw. nicht veranlasste, Eigeninitiative zu entwickeln. Möglicherweise haben Flüchtlinge, denen frühzeitig Aufenthalt gewährt wurde und die sich schnell in den Arbeitsmarkt und die deutsche Umgebung integrieren konnten, derartige Probleme kaum oder gar nicht entwickelt.

Trotz mangelnder Integration, unsicheren Aufenthalts, ungewisser Zukunft, hoher Belastung und finanziellen Anreizen sind die „Push-Faktoren“ nicht stark genug, um sich für eine Rückkehr zu entscheiden, was dem eingangs dargestellten „Push-Pull-Modell“ entspricht.

\section{PTSD-Gruppe}

Es waren über $50.0 \%$ der Befragten von einer PTSD betroffen. Bei ihnen zeigten sich ungünstigere Werte bezüglich Integration, Anzahl komorbider Störungen und subjektiver Lebenszufriedenheit. PTSD-Betroffene zeigten zudem ein signifikant geringeres Interesse an ihrem Herkunftsland und gaben signifikant häufiger die Angst vor Wiedererinnerung an das traumatische Geschehen als Hauptmotiv gegen eine Rückkehr an. Dieses Verhalten könnte im Sinne des Symptomclusters der Vermeidung interpretiert werden, was auch bedeuten würde, dass Flüchtlinge mit PTSD eine Besuchsreise in ihr Herkunftsland vermeiden würden. Tatsächlich waren jedoch die PTSD-Betroffenen, die aufenthaltsrechtlich die Möglichkeit hatten, auch zu Besuch in der Heimat. Inwiefern die PTSD-Vermeidungssymptomatik hier also auf die Gesamtsituation übertragbar ist, bleibt fraglich und bedarf der weiteren Untersuchung.

\section{Fazit}

Es hat sich gezeigt, dass Flüchtlinge aus dem ehemaligen Jugoslawien, die weiterhin in der BRD leben, auch heute noch stark psychisch belastet sind. Als wichtiger Faktor erweisen sich hierbei die traumatischen Erfahrungen, die im Zusammenhang mit dem Krieg gemacht wurden. Dies ist umso relevanter, als sich eine psychische Erkrankung als wichtiges Hindernis für eine Integration im Exil herausstellte. Gleichzeitig bildeten sich affektive Sörungen häufiger bei Personen mit schlechten Integrationswerten heraus.

Derartige Erkenntnisse sollten dazu führen, zukünftig im Umgang mit Flüchtlingen langfristiger und damit verantwortungsvoller zu denken, d. h. bereits bei Aufnahme von Flüchtlingen deren längerfristige Perspektive in den Blick zu nehmen. So sollten asylpolitische Prozesse beschleunigt werden, um mögliche Gesundheitsrisiken beispielsweise durch jahrelanges Warten auf eine Kl ärung der Aufenthaltssituation und die damit verbundene erzwungene Passivität auf Seiten der Flüchtlinge minimiert werden. Die gefundenen Zusammenhänge zwischen psychischer Gesundheit, Integration, subjektiver Lebenszufriedenheit und Rückkehrmotiven zeigen, dass eine mehrdimensionale Herangehensweise an die Thematik notwendig ist, um der Problematik gerecht zu werden und Konsequenzen realistisch einschätzen zu können. Als weiterer Befund ergab sich für die Anbindung an Deutschland die Bedeutung der eigenen Kinder, welche sowohl als „Rückkehrhindernis“, als auch mit ihrer ganz eigenen Problematik in der bisherigen Debatte um ,freiwillige Rückkehr" noch kaum Beachtung finden.

\section{Literatur}

Ackenheil, M., Stotz, G., Dietz-Bauer, R. \& Vossen, A. (1999). Deutsche Fassung des Mini-International Neuropsychiatric Interview. München: Psychiatrische Universitätsklinik München.

Ai, A. L., Peterson, C. \& Ubelhor, D. (2002). War-related trauma and symptoms of Posttraumatic stress disorder among adult Kosovar refugees. Journal of Traumatic Stress, 15(2), $157-160$.

American Psychiatric Association (1980). Diagnostic and statistical Manual of Mental Disorders (3rd ed.). Washington, DC: American Psychiatric Press.

American Psychiatric Association (1994). Diagnostic and statistical Manual of Mental Disorders (4th ed.). Washington, DC: American Psychiatric Press. 
BAGFW (2006). BAGFW Positionspapier zu Bedingungen von freiwilliger Rückkehr von Flüchtlingen. Zugriff am 27.11. 2006. Verfügbar unter: http://www.bagfw.de/?id=927.

BAMF (2006). ZIRF Counselling. Zugriff am 31.1.2007. Verfügbar unter: https://www.zirf.eu.

BAMF (2007). Rechtsgrundlagen. Zugriff am 26.3.2007. Verfügbar unter: http://www.bamf.de/cln_043/nn_566202/DE/ Service/Rechtsgrundlagen.

BMI (2001). Rückkehr von Flüchtlingen. Zugriff am 26.3.2007. Verfügbar unter: http://www.bmi.bund.de.

Dahinden, J. (2005). Asylkonferenz 2005. ,, Rückkehr ins Herkunftsland" - wo funktioniert das Konzept (nicht)? Zugriff am 1.2.2007. Verfügbar unter: http://www.stadtzuerich.ch/ asylkonferenz_2005.

De Jong, J. T. V. M., Komproe, I. H., van Ommeren, M., el Masri, M., Araya, M., Khaled, N., van de Put, W. \& Somasundaram, D. (2001). Lifetime events and posttraumatic stress disorder in 4 post- conflict settings. JAMA, 286 (5), $555-562$.

Ehlers, A., Clark, D. M., Dunmore, E. B., Jaycox, L., Meadows, E. \& Foa, E. B. (1998). Predicting the response to exposure treatment in PTSD. The role of mental defeat and alienation. Journal of Traumatic Stress, 11, 457-471.

Entenmann, T. (2002).Reintegration in Deutschland-politische Entscheidungsträger, Akteure, Programme. Zugriff am 20.6.2006. Verfügbar unter: http:// www.reintegration.net/ deutschland/.

Foa, E. B. (1995).Posttraumatic Stress Diagnostic Scale: Manual. Minneapolis, MN: National Computer Systems.

Gäbel, U., Ruf, M., Schauer, M., Odenwald, M. \& Neuner, F. (2006). Prävalenz der Posttraumatischen Belastungsstörung (PTSD) und Möglichkeiten der Ermittlung in der Asylverfahrenspraxis. Zeitschrift für Klinische Psychologie und Psychotherapie, 35 (1), 12-20.

Gesemann, F. (2006).INTI-Projekt. Indikatoren der Integration im Bildungsbereich. Zugriff am 24.9.2007. Verfügbar unter: http://www.berlin.de/imperia/md/content/lb-integration-migration/themen /eu_projekte/inti_bildung.

Gmelch, G. (1979). Return Migration and Migrant Adjustment in Western Ireland. Irish Found. Hum. Dev., 62, $27 \mathrm{ff}$.

Gmelch, G. (1980). Return Migration. Annual Reviews of Anthropology, 9, 135-59.

Heckmann, F. (2003). From ethnic nation to universalistic immigrant integration: Germany. In F. Heckmann \& D. Schnapper (Eds.), The integration of immigrants in European societies. Stuttgart: Lucius \& Lucius, 45-8.

Herman, J. L. (1994). Die Narben der Gewalt. München: Kindler.

Hunt N. \& Gakenyi, M. (2004). Comparing refugees and nonrefugees: the Bosnian experience. Anxiety Disorders, 19, $717-23$

Johnson, H. \& Thompson, A. (in press). The Development and Maintenance of Post Traumatic Stress Disorder (PTSD) in civilian adult survivors of war trauma and torture: A review, Clinical Psychology Review.

Kivling-Bodén, G. \& Sundbom, E. (2002). The relationship between post-traumatic symptoms and life in exile in a clinical group of refugees from the former YugoslaviaActa Psychiatrica Scandinavica, 105 (6), 461-468.

Kleck, M. (2006). Trauma und Nachkriegszeit. München: Verlag Dr. Hut.

Knipscheer, J. W. \& Kleber, R. J. (2006). The relative contribution of posttraumatic and acculturative stress to subjective mental health among Bosnian refugees. Journal of Clinical Psychology, 62 (3), 339-35.

Lehmann, K.-D. (2006).Psychische Gesundheit und Aufenthaltssituation bei Flüchtlingen. Unveröffentlichte Diplomarbeit, Universität Konstanz.

Lienenkamp, R. (1999). Internationale Wanderungen im 21. Jahrhundert: die Ermittlung von Dispositionsräumen glo- baler Migration auf der Basis von Fuzzy Logic. Dortmund: Dortmunder Vertrieb für Bau- und Planungsliteratur.

Lopes Cardozo, B., Kaiser, R., Gotway, C. A. \& Agani, F. (2003). Mental health, social functioning, and feelings of hatred and revenge, of Kosovar Albanians one year after the war in Kosovo. Journal of Traumatic Stress, 16 (4), 351-360.

Maercker et al. (in press). Posttraumatische Belastungsstörungen in einer Bevölkerungsstichprobe in Deutschland: Vollbild und partielle Störungsbilder. Der Nervenarzt.

Marshall, G. N., Schell, T. L., Elliott, M. N., Berthold, S. \& Chun, C. A. (2005). Mental health of Cambiodian refugees 2 decades after resettlement in the United States.JAMA, 294, 571-579.

Michalowski, I. \& Snel, E. (2005)., Kann man Integration messen?'. Zugriff am 24.9.2007. Verfügbar unter: http://www. muenster.de/stadt/zuwanderung/pdf/2005doku_michalowski-snel.pdf.

Miller, K., Weine, S., Ramic, A., Brkic, N., Smajkic, A., Boskailo, E. \& Worthington, G. (2002). The relative contribution of war experiences and exile- related stressors to levels of psychological distress among Bosnian refugees. Journal of Traumatic Stress, 15 (5), 377-387.

Miller G., Elbert T. \& Rockstroh, B. (2005). Judging psychiatric disorders in refugees. The Lancet, 366, 1604-1605.

Mollica, R. F., Sarajilic, N., Chernoff, M., Lavelle, J., Sarajilic Vukovic, I. \& Massagli, M. P. (2001). Longitudinal study of psychiatric symptoms, disability, mortality, and emigration among Bosnian refugees.JAMA, 286 (5), 546-554.

Powell, S., Rosner, R. \& Butollo, W. (1998). Flight Paths . Munich, Institute for Psychology, University of Munich, $1-41$.

Roth G., Ekblad, S. \& Agren, H. (2006). A longitudinal study of PTSD in a sample of adult mass-evacuated Kosovars, some of whom returned to their home country. European Psychiatry, 21, 152-159.

Schmidt, S., Mühlan, H. \& Power, M. (2006). The EUROHISQOL 8-item index: psychometric results of a cross-cultural field study. The European Journal of Public Health, 16(4), 420-428.

Sheehan D. V., Lecrubier Y., Harnett-Sheehan, K., Amorim P., Janavs, J., Weiller, E., Hergueta, T., Baker, R. \& Dunbar, G. (1998). The Mini International Neuropsychiatric Interview (M.I.N.I.): The Development and Validation of a Structured Diagnostic Psychiatric Interview. J. Clin. Psychiatry, 59 (Suppl. 20), 22-33.

Silove, D., Steel, Z., McGorry, P. \& Mohan, P. (1998). Trauma exposure, postmigration stressors, and symptoms of anxiety, depression and post-traumatic stress in Tamil asylumseekers: comparison with refugees and immigrants Acta Psychiat Scandinavica, 97, 175-181.

Silove, D., Steel, Z. \& Mollica, R. F. (2001). Detention of asylum seekers: Assault on health, human rights, and social development. The Lancet, 357, 1436-1437.

Spitzer R. L., Williams, J. B. W. \& Gibbon, M. et al. (1990). Structured Clinical Interview for DSM-III-R. Washington D.C.: American Psychiatric Press.

Steel, Z., Silove, D., Bird, K., McGorry, P. \& Mohan, P. (1999). Pathways from war-trauma to posttraumatic stress symptoms among Tamil asylum seekers, refugees, and immigrants. Journal of Traumatic Stress, 12 (3), 421-435.

Sundquist J. \& Johansson, S.-E. (1996). The influence of exile and repatriation on mental and physical health.Soc. Psychiatry Psychiatr. Epidemiol., 31, 21-28.

Taylor, E. (1976). The social adjustment of returned migrants to Jamaica. In F. Henry (Ed.), Ethnicity in the Americas. The Hague: Mouton.

Thulesius, H. \& Hakansson, A. (1999). Screening for Posttraumatic stress disorder symptoms among Bosnian refugees. Journal of Traumatic Stress, 12 (1), 167-174.

Toren, N. (1975). The effect of economic incentives on return migration. Int. Migr., 13, 134-144. 
Toscani, L., DeRoo, L. A., Eytan, A., Gex-Fabry, M., Avramovski, V., Loutan, L. \& Bovier, P. (2006). Health status of returnees to Kosovo: Do living conditions during asylum make a difference? Public Health, 596, 1-11.

Weine, S. M., Vojvoda, D., Becker, D. F., McGlashan, T. H., Hodzic, E., Laub, D., Hyman, L., Sawyer, M. \& Lazrove, S. (1998). PTSD symptoms in Bosnian refugees 1 year after resettlement in the United States. American Journal of Psychiatry, 155 (4), 562-564.

WHOQOL Group (1998). Development of the World Health Organization WHOQOL-BREF Quality of Life Assessment. Psychol. Med, 28, 551-558.

World Health Organization (1997). Composite International Diagnostic Interview (CIDI), Version 2.1.Geneva, Switzerland: World Health Organization (WHO).
Yehuda, R. \& McFarlane, A. C. (1995). Conflict between current knowledge about post-traumatic stress disorder and its original conceptual basis.Am J Psychiatry, 152, 1705-1713.

Dipl.-Psych. Ulrike von Lersner

Zentrum für Psychiatrie Reichenau

Psychologische Ambulanz für Flüchtlinge

Feuerleinstraße 55

78479 Reichenau-Linenbrühl

E-Mail: ulrike_vlersner@yahoo.de 